\title{
Clément Juglar, l'Algérie et les colonies
}

\section{Ludovic Frobert}

\section{(2) OpenEdition \\ Journals}

Édition électronique

URL : http://journals.openedition.org/ress/118

DOI : 10.4000/ress. 118

ISSN : 1663-4446

Éditeur

Librairie Droz

Édition imprimée

Date de publication : 1 mars 2009

Pagination : 95-105

ISBN : 978-2-600-01275-1

ISSN : 0048-8046

Référence électronique

Ludovic Frobert, "Clément Juglar, l'Algérie et les colonies », Revue européenne des sciences sociales [En ligne], XLVII-143 | 2009, mis en ligne le 01 mars 2012, consulté le 19 avril 2019. URL : http:// journals.openedition.org/ress/118; DOI : 10.4000/ress.118 


\section{Ludovic FROBERT*}

\section{CLÉMENT JUGLAR, L'ALGÉRIE ET LES COLONIES}

Si les premières mentions chez Clément Juglar des «crises commerciales et de leurs retours périodiques » datent des années 1856-1857, ses tous premiers textes économiques sont légèrement antérieurs. Lors des années 1852-1854, il publie une série de travaux sur la population, sur les équilibres entre industrie et agriculture, et sur l'Algérie et les colonies. Sur ce dernier sujet il fait paraître en 1853 dans le Journal des économistes une série de quatre longs articles ${ }^{1}$. La centaine de pages qu'il consacre à cette question mérite attention pour deux raisons :

- Sur le plan méthodologique ces pages permettent d'observer l'un des outils privilégiés par Juglar lors de ses investigations, le voyage. Le voyage est ici, sur le plan de la méthode, le parent proche de l'enquête ouvrière, un cadre réfléchi d'observations permettant de poser les bases de la théorie.

- Sur le plan doctrinal, les articles sur l'Algérie permettent, une première fois, d'observer le positionnement original de Juglar dans les débats de son temps. Dès ce travail, et alors même que son inscription dans le groupe des économistes de Paris est en cours de réalisation, se développe déjà une idiosyncrasie originale qui va constituer le creuset de ses futures investigations sur la question des cycles économiques.

Après le rappel liminaire de l'état de la question en France autour de 1850 (I), nous nous pencherons sur la fonction méthodologique du voyage chez Juglar (II) avant d'exposer ses arguments doctrinaux sur la colonisation et le cas algérien (III).

\section{QUELS DÉBATS SUR LA COLONISATION EN ALGÉRIE AUTOUR DE 1850?}

Le voyage en Algérie de Clément Juglar a lieu, nous le verrons en détail plus loin, au début de l'année 1852. Il débarque à Alger le 7 février, quelques semaines

ENS-LSH Lyon et CNRS/TRIANGLE. Contact: Ludovic.Frobert@ens-lsh.fr.

1 Clément Juglar, «De la colonisation en Algérie » - $1^{\text {ère }}$ partie: Journal des Economistes, Tome $34,1^{\text {ère }}$ Série, $12^{\mathrm{e}}$ Année, No. 141, 15.01.1853, pp. 40-73. - 2e partie: «Population Européenne, Importations exportations, Population indigène » Journal des Economistes, Tome $35,1^{\text {ère }}$ Série, $12^{\mathrm{e}}$ Année, No. 144, 15 Avril 1853,pp. 80-101.-3 $3^{\mathrm{e}}$ partie: «Corporations indigènes, Finances, Propriété, Concessions », Journal des Economistes, Tome 36, ${ }^{\text {ère }}$ Série, $12^{\mathrm{e}}$ Année, No. 147, 15 Juillet 1853, pp. 89-113. - $4^{\mathrm{e}}$ partie: «Conclusions», Journal des Economistes, Tome 36, $1^{\mathrm{ère}}$ Série, $12^{\mathrm{e}}$ Année, No. 148, 15 Août 1853,pp. 215-233. 
après le coup d'Etat de Louis-Napoléon Bonaparte qui, en France, a mis un terme à trois années de République. Ses observations, ses notes concernent donc d'abord l'œuvre colonisatrice menée par la $2^{\mathrm{e}}$ République entre 1848 et 1851 ; elles reflètent également en grande partie les opinions du lobby libéral qu'il a rejoint au tout début des années 1850 .

Jusqu'à la veille de la Révolution de Février, l'Algérie, sous domination française depuis 1830, avait vécu au rythme de la dictature militaire du «pacha révolté », le général Bugeaud. 1848 et la République furent accueillis avec enthousiasme par les premiers colons qui perçurent là l'occasion de voir cesser ce régime d'exception et de faire de leur territoire une partie de la France ${ }^{2}$. Effectivement, la constitution de novembre 1848 franchit une étape importante en faisant de l'Algérie un «territoire français ». Les trois provinces devinrent de véritables départements, les autorités civiles prétendant concurrencer puis supplanter rapidement dans ces espaces désormais sécurisés, le pouvoir des militaires. Simultanément, les colons pouvaient voir dans le projet de colonies agricoles un renfort de leur espoir d'un peuplement français de l'Algérie. Dès 1843, Barthélémy Prosper Enfantin, qui aura de nombreux relais en Algérie, avait imaginé un modèle de colonisation pacifique et mixte, vecteur d'associations entre colons et indigènes ${ }^{3}$. Plus réaliste, Alexis de Tocqueville avait défendu "la nécessité d'établir une population européenne sur le sol de l'Afrique» pour des raisons de sentiment et de puissance nationaux ${ }^{4}$. En 1848, Pierre Leroux, liant encore grâce au thème de l'association la question des colonies à la question sociale, vantait l'entreprise colonisatrice. A partir de septembre 1848, 16 convois partirent de Paris emportant 13800 colons - ouvriers parisiens au chômage et que ne pouvaient employer les ateliers nationaux - destinés à la construction et au peuplement de 42 villages agricoles. Dans le même temps, le voyage en Algérie de quelques célébrités littéraires, Théophile Gauthier, Edmond et Charles de Gourmont, Louis Veuillot, Alexandre Dumas, leurs récits dans L'Echo de Paris, La Presse ou L'Eclair, facilitèrent encore l'avancée du thème de «l'Algérie française $»^{5}$.

Le caractère longtemps militaire de l'affaire, l'omniprésence de l'Etat, les collusions ambiguës avec certains idéaux socialistes firent de la critique de la colonisation l'un des chevaux de bataille du lobby libéral. C'est en 1852 que Juglar entra à la Société d'économie politique de Paris. Depuis 1848, ces libéraux étaient désormais sur la défensive ${ }^{6}$. Ils étaient la cible des protectionnistes et des industriels proches du pouvoir, aussi bien que des socialistes. Ces années signalent un durcissement net de leur doctrine qui adopte dès lors une tonalité franchement

2 Charles-André Julien, Histoire de l'Algérie contemporaine, tome 1. La conquête et les débuts de la colonisation (1827-1871), Paris, PUF, 1964 ; également, Yvette Katan, «La Seconde République et l'Algérie: une politique de peuplement?», in Jean-Luc Mayaud (dir.), 1848. Actes du colloque du cent cinquantenaire, Paris, CREAPHIS, 2002, pp. 389-412.

3 Barthélemy-Prosper Enfantin, Colonisation de l'Algérie, Bertrand, 1843.

4 Alexis de Tocqueville, «Rapport sur l'Algérie» [1847], in A. de Tocqueville, De la colonie en Algérie, Paris, éditions complexes, 1988.

5 Michèle Salinas, Voyages et voyageurs en Algérie (1830-1930), Privas, 1989.

6 Francis Démier, «Les économistes libéraux et 1848 », in Pierre Dockès et alii. (dir.), Les traditions économiques françaises 1848-1939, Paris, Editions du CNRS, 2000. Lucette Levan-Lemesle, Le juste et le riche : l'enseignement de l'économie politique en France, Paris, 2004. 
conservatrice. Si auparavant, et surtout entre 1840 et 1845, ces libéraux - notamment Adolphe Blanqui - admettaient nombre de préoccupations sociales, après 1848 leurs opinions se figent: arc-boutés sur la doctrine de la conciliation naturelle des intérêts de tous les «industriels », Frédéric Bastiat, Michel Chevalier, Joseph Garnier, Hyppolite Passy et d'autres encore critiquent les différentes formules d'association, de droits, d'organisation du travail. Ils sont rapidement en défaveur des pouvoirs en place et cette situation sera plus nette encore aux tous débuts de l'Empire, ne s'améliorant qu'après 1857.

L'un de leurs principaux terrains polémiques est donc celui de la colonisation, et l'Algérie sert ici systématiquement d'exemple. Leur pamphlétaire vedette, Bastiat, évoque ainsi le sujet en 1850 dans son brûlot contre l'intervention de l'Etat, Ce qu'on voit et ce qu'on ne voit pas dont un des chapitres s'intitule «L'Algérie». Ce que l'on voit, parce que partout on le clame, c'est ce que parvient à réaliser l'Etat grâce à la ponction fiscale. Mais, estime Bastiat, il faut équilibrer le calcul, «de tout le bien attribué à la dépense publique exécutée, il faut donc déduire tout le mal de la dépense privée empêchée», un mal qui bien souvent excède, et de loin, ce bien; en effet, « la présomption est toujours défavorable aux dépenses collectives par voie d'impôt». Les fonctions régaliennes de l'Etat ne portent pas à débat, à la différence de ses fonctions d'allocation des ressources. Ici on ne peut que constater gaspillages et distorsions: "Quand 50 millions sont laissés au contribuable, comme le contribuable est partout, ils alimentent du travail dans les quarante mille communes de France; ils agissent dans le sens d'un lien qui retient chacun sur sa terre natale; ils se répartissent sur tous les travailleurs possibles et sur toutes les industries imaginables. Que si l'Etat, soutirant ces 50 millions aux citoyens, les accumule et les dépenses sur un point donné, il attire sur ce point une quantité proportionnelle de travail déplacé, un nombre correspondant de travailleurs dépaysés, population flottante, déclassée, et j'ose dire dangereuse quand le fond est épuisé » ${ }^{7}$. Les arguments de Bastiat sont repris, classés et développés peu de temps après dans les entrées «Colonies» et «Colonies agricoles » que signe Gustave de Molinari pour le Dictionnaire de l'économie politique de Charles Coquelin. Molinari estime que la colonisation peut se justifier pour les nations à l'étroit et prétendant en outre à mettre en travail des ressources laissées en friche par les «races barbares». Mais il s'agit ici d'une entreprise difficile nécessitant un mélange rare de compétences, de volonté, de responsabilité, et, enfin, de capitaux. On comprend dès lors immédiatement, écrit Molinari, que «le gouvernement ne saurait s'en charger utilement». Or, contrairement au modèle de colonisation commerciale et libre adopté par l'Angleterre, la France a privilégié jusqu'à aujourd'hui le «système colonial», système basé sur le monopole, sur la réglementation, sur les prohibitions; en bref, sur un contrôle strict de l'Etat. Ce système conduit à une mauvaise répartition des ressources et au final, comme le signale le cas de l'Algérie et l'exemple plus précis encore des colonies agricoles, à des résultats économiques désastreux. En Algérie, relève Molinari, les sommes allouées par l'Etat à chaque famille de colon leur auraient

7 Frédéric Bastiat, Ce qu'on voit et ce qu'on ne voit pas, Paris, 1850, chapitre X, «L'Algérie». Ces 50 millions évoqués sont ceux votés par l'Etat français en septembre 1848 pour aider à l'implantation des 42 colonies agricoles en Algérie. 
assuré d'excellentes conditions de vie en France; par ailleurs, les 8000 francs/ famille investis par l'Etat ont «rendu» un bénéfice final moyen de 115 francs/ famille. Ce bilan conduit par contraste à dessiner les traits d'une colonisation réussie: «Le meilleur système à suivre en cette matière, ou, pour mieux dire, le seul bon, c'est de laisser les émigrants aller où bon leur semble, s'établir, se gouverner et se défendre à leur guise et surtout à leurs frais. Leur liberté et leur responsabilité demeurant ainsi entières, ils se rendent, de préférence, dans les endroits où la colonisation présente le plus d'avantages et le moins d'obstacles; ils emploient ainsi les procédés d'exploitation et de gouvernement qui leur semble les plus efficaces et les moins coûteux. Toute protection extérieure, en les exonérant, en partie, de la responsabilité des fautes qu'ils peuvent commettre, encourage la mauvaise distribution et le mauvais emploi de leurs fonds productifs; de même, toute restriction qui les empêche de tirer le meilleur parti possible de leur capital et de leur travail apparaît comme un obstacle au développement de leur

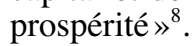

Toutefois, y compris dans le Journal des économistes, la situation n'est pas figée, et force est de constater, même parmi les plus radicalement libéraux, que la colonisation algérienne a enregistré des avancées depuis 1848. Dans les numéros de mai et d'août 1851, Frédéric Lacroix, ancien préfet d'Alger vient présenter quelques idées pratiques pour «activer et développer rapidement la colonisation». Il termine ses articles en détaillant un plan complet de colonisation de l'Algérie, recommandant notamment «d'étendre et fortifier les institutions civiles, élargir le domaine du droit commun ${ }^{9}$. Peu après, J. Cohen signe également un papier optimiste concernant le processus de colonisation en cours ${ }^{10}$. Dans les deux cas, la rédaction de la revue publie une note pour se distancier de ces points de vue, stigmatisant en particulier toute présence excessive de l'Etat dans ce processus: «Mieux vaut pas de colonisation, note ainsi Joseph Garnier, qu'une colonisation artificielle, c'est-à-dire l'établissement de populations et la création d'entreprises qui exigeraient un appareil administratif compliqué, bientôt aussi oppressif pour les colons et aussi ruineux pour la métropole que l'occupation militaire ${ }^{11}$. C'est donc dans ce contexte réactif que Juglar va publier dans le journal une série d'articles extrêmement critiques contre le processus de colonisation en cours.

\section{ASPECTS MÉTHODOLOGIQUES : LES VOYAGES DE JUGLAR}

Le rôle des voyages dans la biographie intellectuelle de Juglar ne doit pas être sous-estimé. Dans la notice que lui consacre son épouse en 1905-1906, il est

${ }^{8}$ Gustave de Molinari, entrées «Colonies» et «Colonies agricoles» dans Charles Coquelin, Dictionnaire de l'économie politique, Paris, Guillaumin, 1852-1853, pp. 393-402 et pp. 402-5.

${ }^{9}$ Frédéric Lacroix, «Quelques idées sur la colonisation en Algérie», Journal des économistes, numéros de mai et août 1851 , p. 18-33 et pp. 136-50.

10 J. Cohen, «L'Algérie vue en beau. Coup d'œil sur le passé et l'avenir de cette colonie », Journal des économistes, septembre 1851, pp. 349-59.

${ }^{11}$ Joseph Garnier, note à la fin de l'article de F. Lacroix, art. cit., p. 150. 
précisé que le tournant entrepris par Juglar de la médecine à l'économie, après 1846, s'explique par plusieurs raisons: impact de la Révolution de février 1848, limites thérapeutiques de la médecine clinique, mais aussi, goût des voyages. Une rapide recension montre que du début des années 1840 , date de son premier voyage en Angleterre, à son décès en 1905, Juglar voyagera une cinquantaine de fois. Le voyage n'est pas ici qu'agrément. Il constitue surtout une expérience privilégiée pour ce médecin de formation, très influencé par le paradigme anatomo-clinique, et donc obnubilé par l'observation réfléchie, par l'observation clinique $^{12}$. Dès 1850 , il visite l'Espagne mais pour en rapporter une enquête statistique sur les hôpitaux espagnols. Deux ans plus tard, il entreprend un voyage de six mois: il passera d'abord un mois et demi en Algérie (février-mars 1852), y reviendra encore en juin, après un passage par l'Italie, la Grèce puis la Turquie, pour visiter la province de Constantine. Les lettres d'introduction qu'il sollicite pour visiter sur place consuls, recteurs, procureurs, soulignent toutes qu'il s'agit d'un «voyage en Orient dans un but scientifique», l'autorisation de séjour du ministère de la guerre précisant même que le voyage du «Docteur Juglar» sera consacré à des «recherches sur la race humaine ${ }^{13}$. Les conditions exactes de son voyage, que l'on peut suivre grâce à son journal, montrent qu'effectivement il ne s'agit pas principalement d'un voyage d'agrément; rappelons d'abord, que lorsque Juglar débarque en Algérie ce n'est que depuis très peu que le pays est ouvert aux voyageurs savants. Longtemps, l'exploration n'a pu être le fait que des militaires qui, dès 1836 publiaient leurs travaux, sous la direction d'Henri Pellissier de Reynaud, dans les Annales Algériennes, où, comme Antoine Carette ou Joseph Daumas allaient faire paraître les premières monographies précises sur le pays; «en Algérie, de 1830 à 1850, comme le souligne aujourd'hui Michèle Salinas, rien n'est définitif; les expéditions sont bien souvent à recommencer; les positions sont mal assurées ». Juglar débarque en Algérie au milieu de l'hiver et décrit des conditions assez rudes. Par ailleurs, il se déplace constamment, visite, observe et prend des notes. Et surtout, il ne demeure pas sur la zone littorale privilégiée alors par les voyageurs mondains: on sait que, vers le sud, il se rend à Médéah [«Route mouillée, sans garde fou, étroite, suit le flanc de la montagne, cahot affreux, fentes effrayantes sur lesquelles la voiture passe. Un éboulement et tout serait perdu», Journal, 3 mars 1852], passe par Blidah, Staouel, Douera. Surtout, il accompagne un convoi militaire vers Boghar, «place forte, porte du sud, dernier poste avancé des français » [Journal, 6 mars 1852]. On sait également, par son journal, que Juglar multiplie les visites, notamment dans les hôpitaux: hôpital civil d'Alger, hôpital du Dey, dispensaire des femmes publiques, hôpital militaire de Blidah... sont chaque fois l'occasion d'observer, prendre des notes, discuter avec les médecins et chirurgiens locaux. Il visite aussi l'établissement agricole des Trappistes à Staouëli, fortement subventionné par l'Etat, et note significativement dans son journal, «ce sont des condamnés militaires et quelques journaliers qui défrichent. Un seul frère vide l'eau d'un fossé. Je n'en vois pas un

${ }^{12}$ Sur cette question voir L. Frobert et O. Hamouda, «...». L'épouse de Juglar rapportera cette phrase d'André Liesse: «C'est d'une série d'observations cliniques sur les crises qu'il a déduit sa loi des crises ».

${ }^{13}$ Tous ces documents se trouvent aux archives familiales. 
seul autre travailler. Il y a 80 frères, 20 pères, une centaine de bêtes à cornes, 1200 moutons pour une concession de 1000 hectares dont 500 sont défrichées» [Journal, 17 février 1852]. Enfin, ce séjour est l'occasion de nombreux passages à la bibliothèque d'Alger ${ }^{14}$.

Le voyage en Algérie sert donc de support au texte que publie Juglar en 1853. Rien d'exceptionnel ici: le voyage est pensé sur le modèle de la «mission économique » que l'Académie des sciences morales et politiques finance régulièrement depuis 1835 et dont un autre médecin hyginiéniste, Louis-René Villermé, avait le premier bénéficié pour rapporter, au terme de son enquête, un célèbre Tableau de l'état physique et moral des ouvriers en France ${ }^{15}$. Le voyage entrepris suivant un plan raisonné commence aussi alors à être décrit, par Frédéric Le Play et ses premiers disciples comme un dispositif majeur de la méthode d'observation ${ }^{16}$.

«De la colonisation en Algérie » s'ouvre par un récit coloré de l'arrivée à Alger. Cette entame a une fonction rhétorique évidente, le texte prétendant ainsi à la véridicité. Le problème, en effet, estime Juglar, est de démonter les discours ambiants, tantôt catastrophistes, tantôt enthousiastes: «je dirai simplement ce que j'ai vu, ce que j'aurai remarqué », note Juglar. Toutefois, dès cette entame narrative, le cadre est posé. L'introduction contraste en effet la vitalité de la nature méditerranéenne des côtes algéroises avec la frénésie artificielle et vaine de la partie européenne de la ville, et la désolation croissante de l'ancienne partie musulmane.

L'étude détaillée qui suit cette entame de l'article fournit alors les éléments «objectifs» de cette évaluation. On entre ici plus nettement dans le registre de l'enquête. Un ensemble large d'observations, prenant le plus souvent, mais pas toujours, la forme de relevés statistiques, permettent de détailler puis d'intégrer dans un seul tableau les différents pans de la réalité observée, démographique, sociologique, économique, ethnologique. Au final 5 chapitres sont abordés par Juglar.

Le premier chapitre concerne différentes mesures de la population européenne en Algérie. Plusieurs constatations négatives en ressortent. Depuis 1830 c'est une population surtout urbaine, qui a peu augmenté et subit, en raison même du volontarisme politique, des fluctuations violentes et artificielles. Au sein de cette population européenne, la proportion de la population française demeure minoritaire; «à de rares intervalles seulement, commente Juglar, la population française, la seule qui puisse nous donner quelques compensations pour tous les sacrifices que l'on fait pour elle, a été en majorité » (55). C'est l'émigration libre, en particulier espagnole, qui a été la plus vigoureuse.

Le second chapitre concerne la répartition économique de cette population. Là encore, le bilan est très défavorable. Juglar rappelle d'abord que l'implantation

${ }^{14}$ Clément Juglar, Journal de voyage, archives familliales.

${ }^{15}$ Louis-René Villermé, Tableau de l'état physique et moral des ouvriers dans les manufactures de coton, de laine et de soie, Paris, J. Renouard, 1840, introduction.

${ }^{16}$ Voir sur ce point notamment les articles ultérieurs d'Urbain Guérin, «La méthode d'observation. L'Utilité des voyages», «La méthode d'observation. La direction des voyages» (La réforme sociale, octobre et novembre 1881) et d'Edmond Demolins, «Les voyageurs de la science sociale» et «L'enseignement de la science sociale et l'école des voyages » (La réforme sociale, août et novembre 1883). 
agricole constitue «le but définitif de toute colonisation» (66) puisqu'il permet à la colonie, distincte ici du comptoir ou du simple poste militaire, de ne plus être entretenue par la métropole: «Partout, note encore Juglar, la proportion dominante de la population agricole, de celle qui est fixée sur le sol, comparée à la population des villes, donne le meilleur signe de stabilité et des garanties d'avenir d'un peuple; c'est même, on peut le dire, le seul caractère auquel on puisse reconnaître une véritable colonie» (66). Or, sur ce terrain, c'est l'échec des différents «plans de colonisation» imaginés par les généraux, de Bugeaud à Lamoricière, qui est sanctionné par les résultats. Dans ces plans, l'Etat n'était pas seulement le protecteur, mais aussi «le créateur, le soutien de ces nouvelles colonies » (69). Au final de ces efforts massifs impulsés par l'Etat, s'il est vrai que la population rurale a légèrement augmenté en Algérie pour atteindre 23800 individus, le rapport à la population européenne totale n'est encore, avec $27 \%$ en 1850 , que très loin du ratio «normal» que l'on constate en métropole.

Le troisième chapitre tente une évaluation de la richesse produite par la colonie algérienne. Juglar constate d'abord les limites de la puissance de production de la population agricole européenne. La culture du froment domine, mais demeure insuffisante pour nourrir l'ensemble de la population; par ailleurs, les expériences d'implantations industrielles, notamment pour le textile (soie et coton) sont décevantes. Ces lacunes sont repérables au niveau de la balance commerciale. Les importations de produits français ont constamment cru avec l'augmentation des effectifs de l'armée. L'étude précise des quatre postes d'échanges entre la métropole et la colonie - farineux alimentaires, animaux vivants, boissons, tissus - signale dans chaque cas un très grave déséquilibre en défaveur de la colonie. Enfin, la colonisation algérienne n'a pas eu l'impact attendu sur la marine française qui ne réalise qu'à peine $50 \%$ du trafic local.

Le quatrième chapitre, plus ethnologique, se penche sur la population indigène, autre «ressource» susceptible de rentabiliser l'entreprise de colonisation. Les mesures ici sont très indécises mais un élément ressort du bilan dressé par Juglar: l'affaiblissement croissant de la population musulmane, décimée par les violences, razzias et déportations menées par l'armée française, affaiblie encore par sa misère grandissante. Un autre élément est souligné par Juglar: le fait que le volontarisme européen a ici désorganisé des structures anciennes efficientes. Il étudie, dans ce sens, les différentes communautés qui, originellement, ont su se diviser le travail. Et il ne cache pas la situation de dépendance dans laquelle se trouve désormais ces anciennes corporations vivantes et industrieuses, vis-à-vis de l'activité des européens. De même, il mène une réflexion similaire sur les problèmes fiscaux. Une colonie doit normalement permettre des entrées fiscales pour la métropole. En Algérie ce n'est pas le cas, les dépenses excèdent très largement les entrées. Deux raisons sont avancées pour expliquer la faiblesse des entrées fiscales provenant des indigènes: d'abord les entraves que la France, jusqu'à 1851, a mis aux exportations de la colonie (droits prohibitifs); ensuite et surtout le démantèlement opéré par les autorités françaises, de l'ancien système fiscal mis en place avant 1830 par le Dey; dès lors, les impôts traditionnels des tribus, l'hokor, l'achour, le zekkat, ont vu leur source se tarir. Là encore l'entreprise coloniale, dirigée par les militaires, est stigmatisée: «Une chose pourra même surprendre, si l'on y réfléchit: c'est qu'après une guerre de destruction et de ruine comme celle que nous avons faite en Afrique depuis vingt ans, on puisse 
encore trouver des populations assez riches pour payer l'impôt. Nos razzias continuelles ont fait disparaître une grande partie des troupeaux. Les tribus, poursuivies et forcées de fuir, ont abandonné la culture de la terre; privées ainsi de moyens d'existence, elles se sont dispersées, non sans perdre un grand nombre des leurs. Quand, par malheur, elles ne se dérobaient pas assez vite à nos coups, une attaque soudaine en avait bientôt raison. La tribu, selon le terme énergique usité en pareille circonstance, était rasée; on ne faisait pas de prisonniers ! C'est ainsi que la population agricole indigène a été peu à peu rejetée du Sahel dans le Tell, et du Tell dans le désert; mais, avant d'y arriver, la tribu était anéantie» (100).

Le dernier chapitre, plus sociologique, concerne l'évolution de la propriété en Algérie. La stabilité des formes de propriété, rappelle Juglar, conditionne la solidité de la «constitution sociale» d'un pays. Deux questions sont alors soulevées dans l'étude de 1853. En premier lieu, Juglar étudie les formes traditionnelles de propriété en Algérie. L'argumentation permet ici de signaler la cohérence et le caractère sagement adaptés des institutions locales, patinées par le temps, expérimentées dans les échanges entre tribus. Il souligne par exemple le caractère astucieux d'un système de propriété urbaine dans lequel on distingue biens melks et biens habous: "Les biens ou propriétés habous sont celles que, dans la crainte d'une confiscation, on a cédées à une mosquée ou à un établissement pieux, sous réserve d'usufruit et à charge de substitution» (105). Ce premier constat sert une nouvelle fois à souligner les ravages opérés par la suite par l'armée française, puis la désorganisation de cette organisation ancienne par les nouvelles directives administratives des colonisateurs. En second lieu, Juglar étudie la politique de concessions menée par l'administration coloniale depuis le début des années 1840 en vue de fixer une classe de cultivateurs sur les terres «libres» algériennes. Or, c'est une suite de mouvements incohérents qu'analyse ici Juglar. L'Etat a rapidement voulu stopper la frénésie spéculative sur ces terres libres. Par une série d'ordonnances restrictives, «l'administration tranchait la plus grande question de la colonisation, à savoir, quel était le genre de culture qui convenait le mieux à l'Algérie: la grande, la moyenne ou la petite. En décidant a priori que c'était la moyenne, et en y soumettant tout concessionnaire, elle a éloigné du même coup tout ceux qui, avec de grands capitaux, auraient voulu tenter la fortune en Afrique» (226).

\section{CAUSES DE L'ÉCHEC DE L'ALGÉRIE FRANÇAISE SELON JUGLAR}

Juglar situe son enquête sous le signe de la démystification. S'opposant aux publicistes ayant convaincu le public français que l'Algérie «est une heureuse colonie où se déverse le trop-plein de la population française, appelée à lui donner un grand développement agricole et industriel» (215), il veut rétablir les faits. Or le constat est double.

Sur le plan économique cette colonisation est un échec. Les indicateurs traditionnels le signalent, notamment un solde de la balance commerciale très défavorable, le montant des exportations algériennes vers la métropole se situant, suivant les années, entre $8 \%$ et $14 \%$ du montant des importations, ces dernières étant, par ailleurs, pour la plus grande part, financées, via les subventions, par la métropole 
elle-même. Par ailleurs, les relevés démographiques confirment amplement cet échec: population décroissante, implantation très majoritairement urbaine, supériorité numérique de l'émigration libre venant d'Espagne et d'Italie.

Sur le plan moral et politique, en dépit de l'abolition récente de l'esclavage, cette colonisation n'a marqué aucun progrès par rapport aux anciennes entreprises de conquêtes. La «fusion des races », la «colonisation mixte», chère aux saintsimoniens et autres réformateurs sociaux, n'ont eu aucune réalité. De fait, souligne Juglar, «nous recommençons en Afrique la même expérience; après avoir tenté d'assimiler la race arabe, de la soumettre aux lois de notre civilisation, il a fallu, en présence de sa répugnance et de sa résistance, avoir recours à des moyens violents. D'abord, on a repoussé les indigènes, puis, inquiété par leurs attaques incessantes, on a été amené à les exterminer» (218-219). Enfin, ce que razzias, pillages et autres expéditions n'ont pas réussi est, au final, réalisé par la domination économique, Juglar évoquant «une «profonde misère indigène... Ils se retirent sans cesse devant nous, nous les remplacerons quand tout aura été anéanti » (221).

Quelles furent en définitive les causes de cet échec? Juglar écarte rapidement deux premiers facteurs. Les conditions naturelles, rudesse du sol et du climat notamment, ne peuvent être sérieusement avancées. Similairement les circonstances historiques de la conquête puis de la première implantation ne sont pas vraiment en cause. Un facteur plus important a été le rôle désastreux joué par l'Etat dans cette entreprise. Tant les pouvoirs civils que militaires ont multiplié les erreurs, signalant, une nouvelle fois, «l'incapacité de l'Etat dans toute entreprise industrielle ou agricole» (223). L'Etat a failli aussi bien en tant qu'entrepreneur, les colonies agricoles n'ayant donné que des résultats très médiocres, qu'en tant que législateur, les différentes ordonnances sur propriété et concession se signalant par leur arbitraire et leur caractère inadapté à la situation locale. En définitive, «bien rarement, les dispositions prises par l'administration ont atteint le but que l'on se proposait, heureuses encore, quand elle n'ont pas eu un effet tout contraire» (231).

Pourtant la véritable cause de l'échec français en Algérie se situe encore ailleurs. C'est ici qu'apparaît l'argumentation originale de Juglar: cet échec reflète simplement la bonne situation économique de la France; "pour moi, écritil, je ne connais pas de témoignage plus éclatant des bonnes conditions de l'état social et de la civilisation en France, que les difficultés, pour ne pas dire, les impossibilités que nous éprouvons à peupler et coloniser l'Algérie» (231). Les capitaux et surtout les hommes ne se sont pas déplacés pour cette raison. Ce point est encore mentionné précisément à propos des populations: «Si notre population ne cherche pas plus à émigrer avec les facilités, les subventions qu'on lui offre, c'est que son existence est assez bien assurée en France, sans qu'elle éprouve le besoin de changer de pays pour l'améliorer» (232).

Les textes que Juglar consacre à l'Algérie parlent ainsi surtout de la France au lendemain de 1848 et de trois années de République. Ces textes présentent deux acquis doctrinaux majeurs, qui vont constituer trois ans plus tard le creuset de sa vision des $«$ crises commerciales et de leurs retours périodiques ${ }^{17}$ : en premier

${ }^{17}$ Clément Juglar, «Des crises commerciales», Annuaire de l'économie politique et de la statistique, Paris, Guillaumin, 1856, pp. 555-581; «Des crises commerciales et monétaires de 1800 à 
lieu, les sociétés européennes sont désormais animées par des forces industrielles: conquêtes ou révolutions ne sont que des accidents à la surface de phénomènes plus fondamentaux. L'échec du volontarisme colonial en Algérie est ici exemplaire; en second lieu, ces nouvelles forces industrielles manifestent une propriété singulière: elles permettent une croissance de ces sociétés nouvelles, mais par l'intermédiaire de fluctuations régulières. Il s'agit là d'une propriété naturelle que la sagesse politique doit apprendre à respecter. Le thème du progrès apparaît distinctement dans la conclusion iconoclaste de l'étude de 1853: la colonisation a avorté car l'économie française est globalement en progression et que les principaux agents n'avaient aucun intérêt à tenter l'aventure algérienne. Celui du progrès par fluctuation est lui déjà visible dans l'étude légèrement antérieure que Juglar a consacré à l'évolution de la population française dans la première moitié du $19^{\mathrm{e}}$ siècle. Dans ce travail, il repère déjà en effet un "mouvement de hausse et de baisse [...] une soupape de sécurité employée par la Providence pour modérer la marche croissante des peuples ». Mais on trouve indirectement trace de ce second thème dans la série d'articles de 1853. En effet, l'idée de périodicité permet de mieux comprendre l'échec total du tournant colonisateur pris par la $2^{\mathrm{e}}$ République. Au départ ce tournant fut pris, estime Juglar, en raison d'un diagnostic erroné: une France jugée trop peuplée, proche de la stagnation, et dès lors en excès de bras ouvriers qu'il devenait nécessaire de déverser sur des territoires nouveaux. Grâce à sa théorie du progrès par retours périodiques, Juglar diagnostique, au contraire, que loin de constituer les indices d'une tendance lourde, les évènements de février 1848 en France, n'étaient que les signes d'un retournement normal de conjoncture économique en 1846-1847. Que dès lors, tout le volontarisme s'étant exprimé dans l'œuvre colonisatrice en Algérie reconnaissance comme territoire français, émigration et création des colonies agricoles -, était hors de propos, démesuré, dans la mesure où, naturellement, quelques années plus tard, sans intervention humaine, les indicateurs économiques allaient repartir à la hausse, et les bras en excédent trouver preneur.

$$
* * *
$$

Clément Juglar évoquera encore, par la suite, à quelques reprises cette question des colonies. Sans y consacrer de nouveaux articles, il interviendra lors de séances de l'académie des sciences morales et politiques ou de la société d'économie politique de Paris, notamment en 1869, en 1881 et en 1898. A nouveau, il montrera que l'entreprise colonisatrice (si l'on excepte le cas des comptoirs) ne peut être que violente et brutaliser des pans entiers de la réalité politique, sociale, économique, culturelle. Il ajoutera toutefois un argument nouveau. Si l'entreprise est tentée, elle ne peut, au risque d'échouer, surtout lors de ses premières phases de croissance, s'accommoder d'un contexte politique démocratique. Cet argument est développé en 1881. La France présente-t-elle les conditions d'une nation colonisatrice, soucieuse de s'étendre et de renforcer son

$1857 »$, Journal des économistes, $2^{\mathrm{e}}$ série, vol. 14 , n40, avril 1857, pp. 35-60 et $\mathrm{n}^{\circ} 41$, mai 1857 , pp. 255-267; Des crises commerciales et de leur retour périodique en France, en Angleterre et aux Etats-Unis, Paris, Guillaumin, 1862. 
commerce? Deux conditions sont indispensables : une population en excédent par rapport aux ressources, et un Etat susceptible d'imposer un régime de privilèges en faveur des émigrants pour favoriser leur implantation, encourager leurs premiers efforts, protéger leurs résultats. Or, selon Juglar, la France ne vérifie aucune de ces deux conditions. Il l'a déjà dit concernant la première dans l'étude de 1853 et le répète quelques trente années plus tard: «les conditions de l'existence sont tellement douces en France qu'on ne sent pas le besoin d'émigrer ${ }^{18}$. Mais Juglar ajoute également que l'Etat n'est pas en mesure en France d'imposer une véritable politique de colonisation en raison même de ses valeurs politiques: la France, en effet, est «un pays où le sentiment de l'égalité domine tous les autres ». Or, cette option démocratique nuit à la croissance, ce que signale Juglar par le biais d'un exemple tiré de l'histoire: «Observons les grands peuples colonisateurs, la Hollande, l'Angleterre, l'Espagne, partout nous trouvons la trace d'une législation exceptionnelle; l'esclavage n'a disparu que de nos jours, et longtemps après la période de formation, la richesse s'était déjà assez développée pour résister à la secousse de l'émancipation». Si les textes que Juglar consacre aux colonies nous renseignent donc sur sa méthodologie ainsi que sur certaines composantes de sa vision des «crises et de leur retour périodique», ils nous montrent aussi un économiste qui au gré des allers et retours de l'idée républicaine en France dans la seconde moitié du $19^{\mathrm{e}}$ siècle, ne cesse de réfléchir / dans son œuvre à l'articulation problématique entre économie et politique, entre croissance et démocratie.

${ }^{18}$ Clément Juglar, intervention à la séance de la Société d'économie politique du 5 décembre 1881, «De l'illusion des colonies », Journal des économistes, décembre 1881, pp. 476 et 477. 